\title{
Patient decision-making in symptomatic gallbladder disease
}

\author{
Alex Lois $^{1}\left(\mathbb{D} \cdot\right.$ David Droullard $^{1} \cdot$ Jenney Lee ${ }^{2} \cdot$ David Flum $^{1,3,4}$ \\ Received: 3 June 2021 / Accepted: 7 February 2022 / Published online: 1 March 2022 \\ (c) The Author(s), under exclusive licence to Springer Science+Business Media, LLC, part of Springer Nature 2022
}

\begin{abstract}
Background One in five people with gallstones develop symptoms ( 13 million in the US), yet only a fraction undergo cholecystectomy. The frequency of and factors associated with patients choosing medical management for gallbladder disease are not well understood. The goal of this study was to assess patient decision-making regarding cholecystectomy, experiences with medical management, and interest in a clinical trial comparing cholecystectomy with medical management.

Methods We conducted a cross-sectional survey from January 2020 through May 2020 using Amazon's crowdsourcing platform, MTurk. After a brief screening survey to determine gallbladder disease history and cholecystectomy status, eligible users without prior cholecystectomy were invited to complete the full survey for US $\$ 2.50$.

Results There were 148 responses included in analysis (93.7\% of attempted). Participants were $54 \%$ female with a mean age of 36.2 (SD 11.3) years. 36\% of participants had used medications or supplements to manage their symptoms with $17 \%$ using lecithin and $22 \%$ using ursodeoxycholic acid (Ursodiol ${ }^{\circledR}$ ). $85 \%$ attempted lifestyle modifications to manage symptoms. Surgery was recommended for $60 \%$ of which $43 \%$ reported planning cholecystectomy. Among those recommended operation, most common reasons for pursuing were symptoms (50\%) and symptoms interfering with care of family (42\%), while most common reasons for not pursuing were risks of surgery/anesthesia (56\%), ongoing medical management (50\%), and symptom tolerance (38\%). $67 \%$ reported willingness to participate in a clinical trial comparing medical management to cholecystectomy.

Conclusion A significant portion of patients with symptomatic gallbladder disease choose not to undergo cholecystectomy. One of the most common reasons for avoiding surgery was the use of alternative therapies, despite limited evidence to support their use. The lack of prospective comparison of cholecystectomy to medical therapies for the management of gallbladder disease, along with patient interest, support the need for a clinical trial.
\end{abstract}

Gallbladder disease is common in Western populations, with gallstones prevalent in one in five adults [1]. Of those with gallstones, it is estimated that one-fifth $(\sim 13$ million people

Accepted as a podium presentation titled "Patient Preferences in Management of Symptomatic Gallbladder Disease" at SAGES 2021, Las Vegas, NV, August 2021.

Alex Lois

alexlois@uw.edu

1 Department of Surgery, Surgical Outcomes Research Center, University of Washington, 1107 NE 45th Street, Suite 502, UW Box 354808, Seattle, WA 98105, USA

2 Department of Urology, University of Washington, Seattle, WA, USA

3 Department of Health Services, University of Washington, Seattle, WA, USA

4 Department of Pharmacy, University of Washington, Seattle, WA, USA in the United States [US]) will develop symptoms in their lifetime [2]. As a result, laparoscopic cholecystectomy (LC) is one of the most common abdominal surgeries performed in the US, with as many as 700,000 undergoing the procedure annually [3]. However, the discrepancy between the number of patients presenting with symptomatic gallbladder disease-estimated 1.8 million ambulatory care visits each year-and the number cholecystectomies performed suggests that a significant number of patients are foregoing or delaying cholecystectomy in lieu of alternative management strategies $[4,5]$.

A number of medical therapies for gallstones have been proposed but few have demonstrated meaningful efficacy [6]. These include oral supplements, such as lecithin, a synthetic phospholipid; and oral medications, such as ursodeoxycholic acid (Ursodiol $®$ ), a synthetic bile acid, and cholesterol-lowering medications (e.g., Simvastatin®) [7]. As a result of the limited evidence, physicians are left with few alternatives 
to offer patients hesitant to undergo LC. However, despite the lack of level one evidence to support these therapies, a number of non-peer-reviewed sources continue to recommend their use [8-10]. The frequency with which patients are turning to these therapies is not known.

The factors associated with patients choosing medical management over cholecystectomy for gallbladder disease have not been previously described. Understanding this decision-making process would allow surgeons to better counsel patients in the management of their gallbladder disease. The goal of this study was to examine the factors in decisionmaking for patients with symptomatic gallbladder disease among patients that have not undergone cholecystectomy using Amazon's Mechanical Turk (MTurk: Amazon, Inc.), a crowdsourcing platform. As a secondary goal, we assessed the use of medical management strategies and interest in a clinical trial comparing medical and surgical management of symptomatic gallstone disease.

\section{Methods}

\section{Study design}

We conducted a cross-sectional survey using MTurk from January through May 2020, targeting US adults (age 18 and older) with a history of gallbladder disease who had not previously undergone cholecystectomy. MTurk is an online labor market consisting of tens of thousands of workers who can be recruited to complete Human Intelligence Tasks (HITs). The questionnaire administered in this study was developed according to the CHERRIES guidelines for Webbased surveys [11]. Participants were provided information on the purpose of the study, data that would be collected, time to complete the questionnaire, and reimbursement prior to agreeing to participate. The questionnaire was designed using the Qualtrics (Provo, UT) survey platform by our multidisciplinary team. This study was deemed exempt from review by the University of Washington Human Subjects Division (STUDY00008920) prior to survey administration.

\section{Study population}

MTurk users residing in the US with at least 100 prior completed HITs and a HIT approval percentage greater than 95\% were eligible to complete the screening questionnaire which assessed personal history of gallbladder disease and prior cholecystectomy $[12,13]$. The purpose of the screening questionnaire was to ensure participants met the study inclusion criteria of a history of gallbladder disease without prior cholecystectomy. The internet protocol (IP) addresses associated with each response was recorded in order to identify and remove IP addresses providing multiple or inconsistent responses. The questionnaires incorporated reCAPTCHA to prevent internet bots from accessing the survey. Users with a history of benign gallbladder disease that had not previously undergone cholecystectomy were invited to complete the password-protected, full questionnaire assessing their experience. Participants were reimbursed \$2.50 USD based on the time required to complete each survey and the Washington state minimum wage ( $\$ 13.50 \mathrm{USD} / \mathrm{hr})$ at the time of survey administration [14].

\section{Questionnaire structure}

The full questionnaire consisted of 47 questions, requiring approximately $10 \mathrm{~min}$ to complete. First, participants were asked to describe their gallbladder disease (i.e., cholelithiasis, cholecystitis, dyskinesia), whether cholecystectomy was recommended by a surgeon, plans for future treatment (i.e., future surgery), and reasons for or against choosing cholecystectomy. Next, participants were asked about factors in their decision-making regarding treatment for their gallbladder disease. Participants were asked to rate the influence of 14 factors (Fig. 1) in their decision-making using a Likert scale from 1 "Not at all important" to 5 "Extremely important." These factors were based on previous studies of patient-centered outcomes in biliary disease [15]. Free response questions allowed participants to provide additional factors in their decision-making that were not previously listed.

Participants were then asked about their experience with medicinal interventions for gallbladder disease, including lecithin, ursodeoxycholic acid, statin medications, and diet and lifestyle modifications. A description was provided for each medication that included each medication's mechanism of action, generic and trade names for each, and how each medication is typically used. Questions were then posed assessing prior use, interest in future use, and willingness to participate in a hypothetical-randomized trial comparing

\begin{tabular}{l}
\hline Decision-Making Factors \\
\hline Frequency/severity of pain attacks \\
Dietary restrictions \\
Other bowel symptoms \\
Ability to miss work \\
Ability to care for family \\
Physical limitations post-surgery \\
Risks of surgery \\
Fear of surgery \\
Scarring after surgery \\
Cost of surgery \\
Physician recommendation \\
Other recommendation \\
Research supporting treatment \\
Advertising for treatment \\
\hline
\end{tabular}

Fig. 1 Pre-specified decision-making factors 
medical management to cholecystectomy for symptomatic gallbladder disease. Additional free response questions assessed reasons for/against willingness to participate this hypothetical trial. Current gastrointestinal quality of life (GI QoL) was assessed using the 10-question gastrointestinal quality of life index (GIQLI-10) to understand the role of ongoing symptom in decision-making. The GIQLI-10, an abbreviated version of the of the 36-question gastrointestinal quality of life index (GIQLI), assesses gastrointestinal symptoms in the previous two weeks and is scored from 0 "low" to 40 "high" [16-18]. Prior to completion of the survey participants were asked to provide their age, sex, gender identity, race, ethnicity, highest education level achieved, and source of medical insurance. Two consistency checks were built into the full questionnaire, to ensure participant attention and consistency.

\section{Data analysis}

Data analysis was conducted using Stata 16.1 (College Station, TX). Individuals who failed consistency checks were excluded from analysis. Categorical data were compared using chi-squared tests and Fisher's exact test where appropriate. Normally distributed continuous data were summarized as mean and standard deviation (SD) and compared using a student's $t$ test. Factors in decision-making were compared between participants planning and not planning cholecystectomy, limited to individuals for whom a surgeon offered or recommended surgery. The relative importance of each factor was determined comparing the average score of each factor to the composite average of all factors. Thematic analysis of free response questions was performed by two authors (AL and JL) to identify additional factors influencing patient decision-making. Complete case analysis was performed as missing data were minimal and unlikely to introduce bias.

\section{Results}

\section{Study population}

The screening survey received 1011 responses. Duplicate and inconsistent responses to screening were eliminated leaving 859 quality responses (Fig. 2). Of these, 219 were eligible to complete the full questionnaire resulting in 153 responses. Five surveys were excluded based on ineligible responses (no gallbladder disease $[n=2]$, prior cholecystectomy $[n=1]$, low-quality responses $[n=1]$, and failed consistency check $[n=1])$. The final study population of 148 participants was $54 \%$ female with a mean age of 36 (SD 12) years. Most described their disease as symptomatic gallstones (57\%), followed by cholecystitis (26\%) and then

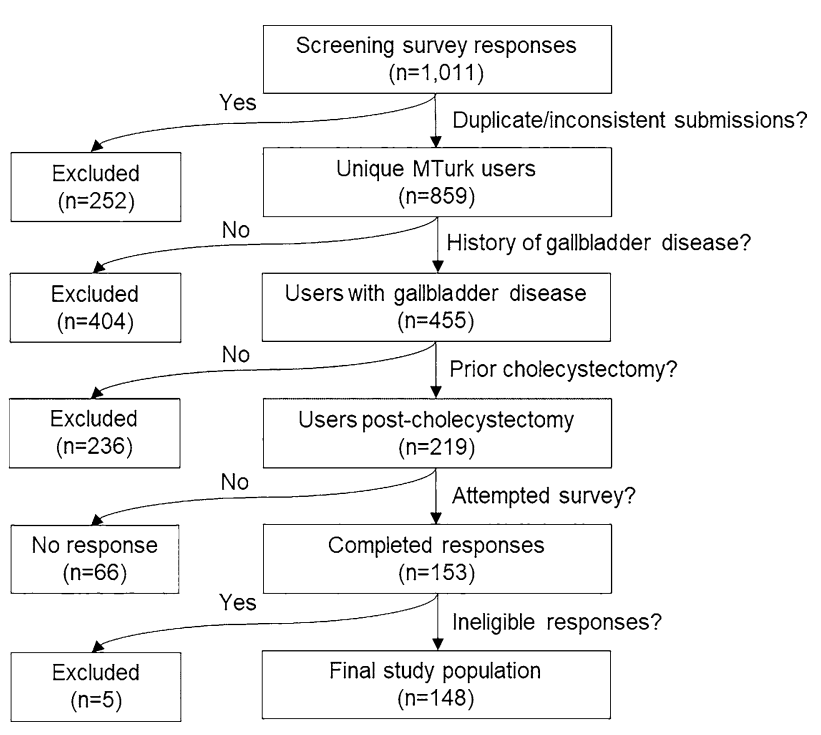

Fig. 2 Arrival at study population

dyskinesia (17\%). The majority $(81 \%)$ reported symptom onset at least six months prior to survey completion.

\section{Planned cholecystectomy}

Approximately one-third of participants (50/148) reported planning cholecystectomy in the future. When directly asked their reasons for pursuing surgery, the most common responses were frequency/severity of symptoms (50\%), inability to care for family due to symptoms (42\%), and worry about future attacks (41\%). In those not planning surgery, the most cited reasons included risks of surgery and anesthesia (56\%), use of other therapies (50\%), and current tolerance of symptoms (38\%). Only $6 \%$ of participants not pursuing surgery cited a lack of benefit (i.e., "I don't think surgery would help my symptoms") as a reason for their decision. GI QoL was lower in those planning surgery (32.4 [95\% Confidence Interval (CI) 30.5-34.3] vs 36.2 [34.8-37.6]). Characteristics of participants planning and not planning to have surgery are described in Table 1.

\section{Factors in decision-making}

Among those planning cholecystectomy, 38 (76\%) reported surgery was recommended by a surgeon compared to $50(51 \%)$ of those not planning for surgery. Among all patients recommend surgery, factors rated as most important in their decision were the frequency/severity of pain symptoms, cost of surgery, and inability to miss work for surgery. Scarring, recommendations of non-physicians, and advertising did not factor heavily in participant decision-making. Compared to participants not planning cholecystectomy, participants planning for surgery rated 
Table 1 Participant characteristics $(n=148)$

\begin{tabular}{|c|c|c|c|}
\hline & $\begin{array}{l}\text { Cholecystectomy } \\
\text { planned }(n=50)\end{array}$ & $\begin{array}{l}\text { Cholecystectomy not } \\
\text { planned }(n=98)\end{array}$ & $p$ Value \\
\hline Age & & & 0.83 \\
\hline Mean (SD) & $35.7(12.6)$ & $36.1(11.1)$ & \\
\hline Sex, $n(\%)$ & & & 0.01 \\
\hline Female & $19(38)$ & $61(62)$ & \\
\hline Race, $n(\%)$ & & & 0.09 \\
\hline White & $34(68)$ & $82(84)$ & \\
\hline Black & $6(12)$ & $7(7)$ & \\
\hline Asian & $1(2)$ & $0(0)$ & \\
\hline Native American or Alaska Native & $6(12)$ & $2(2)$ & \\
\hline Hawaiian Native or Pacific Islander & $1(2)$ & $0(0)$ & \\
\hline Multiracial & $2(4)$ & $7(7)$ & \\
\hline Ethnicity, $n(\%)$ & & & 0.01 \\
\hline Hispanic/Latinx/Spanish & $12(24)$ & $8(8)$ & \\
\hline Education level, $n(\%)$ & & & 0.45 \\
\hline High school diploma/GED & $5(10)$ & $6(6)$ & \\
\hline Some college & $8(16)$ & $29(30)$ & \\
\hline 2-Year degree & $4(8)$ & $8(8)$ & \\
\hline 4-Year degree & $27(54)$ & $41(42)$ & \\
\hline Graduate degree & $6(12)$ & $14(14)$ & \\
\hline Source of insurance, $n(\%)$ & & & 0.03 \\
\hline Employer based & $21(42)$ & $55(56)$ & \\
\hline Purchased plan & $8(16)$ & $7(7)$ & \\
\hline Medicare & $12(24)$ & $7(7)$ & \\
\hline Medicaid & $5(10)$ & $10(10)$ & \\
\hline Other* & $0(0)$ & $9(9)$ & \\
\hline None & $4(8)$ & $10(10)$ & \\
\hline Disease description, $n(\%)$ & & & 0.69 \\
\hline Symptomatic cholelithiasis & $31(62)$ & $54(55)$ & \\
\hline Cholecystitis & $12(24)$ & $26(27)$ & \\
\hline Biliary dyskinesia & $7(14)$ & $18(18)$ & \\
\hline Time since symptom onset, $n(\%)$ & & & 0.15 \\
\hline Less than 6 months & $9(18)$ & 19 (19) & \\
\hline 6 Months to 1 year & $15(30)$ & $21(21)$ & \\
\hline 1 Year to 2 years & $16(32)$ & $26(27)$ & \\
\hline 2 Years to 5 years & $9(18)$ & $17(17)$ & \\
\hline More than 5 years & $1(2)$ & $15(15)$ & \\
\hline
\end{tabular}

*Other includes Military/VA, Indian Health Services, and other non-specified sources of insurance physician recommendations and research supporting treatment as more important and cost as less important in their decision (Fig. 3). Analysis of free responses identified several additional recurring themes in decision-making. Several participants cited their current quality of life, as well as that after surgery, as a driving factor in their decision. Additionally, $10 \%$ of those not planning surgery cited their use of alternative therapies as their primary reason. Of note, one participant cited the COVID-19 pandemic as their reason for not planning surgery.

\section{Experience with medical management}

Use of alternative therapies was high in the study population with $36 \%$ reporting prior or ongoing use of supplements or medications for their gallbladder symptoms. Prior lecithin use was reported by $17 \%$ and $71 \%$ of participants reported willingness to take lecithin if recommended by a physician. Ursodeoxycholic acid use was reported by $22 \%$, and $58 \%$ would be willing to try it after explanation of potential side effects. Statin use was more 
Fig. 3 Relative importance of factors in decision-making among patients recommended cholecystectomy. Decisionmaking factors for treatment of gallbladder disease among patients recommended surgery. Scored on Likert scale from 1 "Not at all important" to 5 "Extremely important." Presented as mean categorical response with axis set at cumulative mean response of 3.25 .

* Indicates $p$ value $<0.05$ from two-sided $t$ test

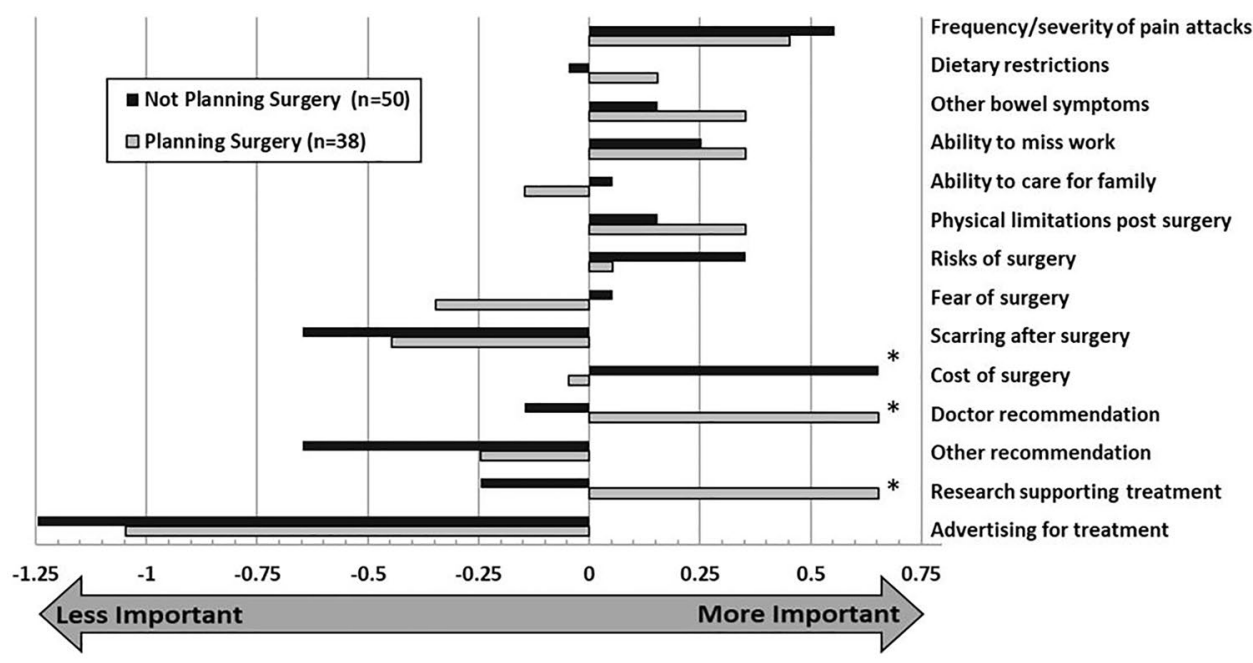

common, reported by $30 \%$ with $56 \%$ willing to trial for their gallbladder. Thematic analysis of responses provided by those unwilling to trial medical therapies identified concerns about side effects, lack of evidence, and distrust of medications as the most common reasons for refusal. Diet and lifestyle modifications had been attempted by $85 \%$ and $91 \%$ reported they would be willing to attempt lifestyle modifications as part of clinical research study. Analysis of write-in responses identified non-specific "gallbladder flushes" as the only additional non-surgical therapy used by participants.

\section{Interest in a clinical trial}

Willingness to participate in a randomized trial comparing medical and surgical management of gallbladder disease was high with $67 \%$ reporting they would be open to participating in this hypothetical trial. Participants provided a wide range of reasons for their interest in a clinical trial. From thematic analysis, the most common themes provided included avoidance of surgery (e.g., "Surgery is invasive and could put me out for weeks..."), desire for relief from ongoing symptoms (e.g., "I am desperate for relief'), and contributing to research that could help others (e.g., "Anything that would help research towards gallbladder disease"). Among those who would not participate in a clinical trial, $41 \%$ described a preference to avoid randomization (e.g., "I just want the decision to be mine" and "I enjoy my freedom of choice"). Several participants specifically cited the possibility being assigned to surgery as their reason for not participating (e.g., "I have no interest in having surgery"), while others cited the burdens of participation (e.g., "Too much work" and "[I don't] have the time to set aside for such trials").

\section{Discussion}

To the best of our knowledge this is the first study to assess factors in treatment decision-making among individuals with gallbladder disease. The majority of participants in this study had been offered surgery $(60 \%)$, yet less than half of these individuals (43\%) reported planning cholecystectomy in the future. Quality of life impairment factors heavily in this decision as evidenced by the frequency and severity of symptoms being the most common reason provided by both those pursuing and not pursuing surgery. This is further evidenced by the lower GIQLI-10 scores observed in individuals planning for surgery. The use of medical management in this cohort was high with more than one-third reporting previous experiences with an oral supplement or medication for their gallbladder. Further, ongoing medical management was one of the most common reasons provided among those not considering surgery. In the context of the frequency with which participants report turning to medical therapies for their gallbladder, it is not surprising that interest in a clinical trial to inform the use of these therapies was high.

Shared decision-making is critical to ensure treatment decisions align with patients' values and understanding the factors influencing patient decision-making is necessary in this process [19]. One prior study found that perceived autonomy and effective surgeon communication were as important as expected postoperative quality of life in patients' decisions to undergo cholecystectomy [20]. In this cohort of US adults with gallbladder disease we show that after severity of symptoms, cost of surgery and time off from work were the most important factors in considering cholecystectomy. These findings are consistent with past studies of decision-making factors for patients undergoing elective surgery. Prior studies of elective orthopedic surgery found quality of life and cost of surgery as principal factors in the decision-making process. [21, 22] However, few 
surgeons discuss cost with their patients. Only $7 \%$ of patients who underwent elective hand surgery reported discussing cost of surgery with their surgeon, despite $48 \%$ feeling doctors should initiate a conversation about cost of care and $59 \%$ agreeing that surgeons should consider costs to patients when making treatment decisions [23]. However, it must also be acknowledged that the relative importance of decision-making factors differ from patient to patient and that there is no "one size fits all" approach to shared decisionmaking [24]. In our study population, patients considering surgery were less concerned about cost of surgery and more highly valued physician recommendation and research supporting the treatment than those not considering surgery.

We identified a high use of medical therapies in this population-one of the most frequently cited reasons for not pursuing cholecystectomy in our study. It is clear that there is strong interest among patients for more studies of alternatives to cholecystectomy of symptomatic gallstones disease. Yet, there are a paucity of prospective comparisons of medical management to surgery in symptomatic gallbladder disease [5]. In addition to patient interest, there is a strong clinical need to address this evidence gap. It is recognized that a significant portion of patients continue to experience symptoms after cholecystectomy [25-27]. Several studies have also demonstrated no significant difference in pain outcomes and satisfaction between patients who do and do not undergo cholecystectomy for symptomatic gallstones [28, 29]. These studies point to the need to refine the surgical selection process. Identifying evidence-based alternatives to cholecystectomy could greatly improve the care of patients with QoL-limiting disease who prefer to avoid surgery or are unlikely to improve with cholecystectomy.

This study has several limitations. These findings may not be generalizable to entire population with gallbladder disease given the small sample size and that the MTurk population tends to be younger, more often male, less racially diverse, and more educated than the general US population. However, MTurk has been shown to be more representative than other convenience samples typically used in survey research [30]. Limiting the questionnaire to participants who had not had prior cholecystectomy likely selected for individuals with less severe symptoms and disease. However, patients with complicated gallstone disease (i.e., choledocholithiasis, gallstone pancreatitis) would not be candidates for medical management and were not relevant to our research question. This study relies on participant-reported data and lacked clinical information, therefore we could not adjust for severity of disease that may have impacted patient decision-making around surgery. However, we expect that most patients with more severe disease would have previously undergone cholecystectomy and thus been excluded from the study. Further, gallbladder disease type did not differ between those planning and not planning surgery. Some have voiced concerns about data quality from MTurk and we addressed these data quality concerns using recommended user quality thresholds, using methods to detect duplicate responses, including a screening survey, and including questions to monitor internal consistency [13]. Finally, our questionnaire assessed participants' stated rather than revealed preferences and it is possible that a number of participants who indicated willingness to participate in a clinical trial would decline if approached. Despite these limitations, our findings describe the relative importance of decision-making factors in cholecystectomy, as well as, the frequency of medical management for gallbladder disease.

These results provide important insight into the decisionmaking of individuals with symptomatic gallbladder disease. Quality of life and the financial consequences of elective surgery are significant factors in this decision and should be explicitly addressed by surgeons. A significant number of patients opt for medical management options instead of cholecystectomy for their gallbladder disease despite a paucity of evidence supporting these treatments. The ongoing use of these therapies and substantial patient interest support the need for a clinical trial comparing their effectiveness with surgery. The results of such a trial, combined with an improved understanding of patient decision-making factors, could greatly improve patient-centered care for gallbladder disease.

Acknowledgements This work is supported by a training grant from the National Institute of Diabetes and Digestive and Kidney Diseases of the National Institutes of Health under award number T32DK070555.

\section{Declarations}

Disclosures Dr. Alex Lois, Dr. David Droullard, Dr. David Flum, and Jenney Lee have no conflicts of interest or financial ties to disclose.

\section{References}

1. Aerts R, Penninckx F (2003) The burden of gallstone disease in Europe. Aliment Pharmacol Ther 18(Suppl 3):49-53

2. Lammert F, Gurusamy K, Ko CW, Miquel JF, Méndez-Sánchez N, Portincasa P, van Erpecum KJ, van Laarhoven CJ, Wang DQ (2016) Gallstones. Nat Rev Dis Primers 2:16024

3. Urbach DR, Stukel TA (2005) Rate of elective cholecystectomy and the incidence of severe gallstone disease. Can Med Assoc J 172(8):1015-1019

4. Shaheen NJ, Hansen RA, Morgan DR, Gangarosa LM, Ringel Y, Thiny MT, Russo MW, Sandler RS (2006) The burden of gastrointestinal and liver diseases, 2006. Am J Gastroenterol 101(9):2128-2138

5. Stinton LM, Shaffer EA (2012) Epidemiology of gallbladder disease: cholelithiasis and cancer. Gut Liver 6(2):172-187

6. Abraham S, Rivero HG, Erlikh IV, Griffith LF, Kondamudi VK (2014) Surgical and nonsurgical management of gallstones. Am Fam Physician 89(10):795-802 
7. Lois A, Hantouli MN, Davidson GH, Flum DR (2020) Medical management of symptomatic gallstones: a narrative review. J Surg Oper Care 5(1):105

8. Khatri M (2021) Understanding gallstones - prevention. Available at https://www.webmd.com. April 22, 2021. Accessed 27 May 2021

9. Avino M (2018) Avoiding gallbladder attack-lecithin may help you reduce and eliminate gallstones. Available at https://docto reden.org. Accessed 27 May 2021

10. Carballido E (2019) Lecithin for gallstones. Available at https:// www.botanical-online.com. April 22, 2019. Accessed 27 May 2021

11 Eysenbach G (2004) Improving the quality of Web surveys: the Checklist for Reporting Results of Internet E-Surveys (CHERRIES). J Med Internet Res 6(3):e34

12. Robinson J, Rosenzweig C, Moss AJ, Litman L (2019) Tapped out or barely tapped? Recommendations for how to harness the vast and largely unused potential of the Mechanical Turk participant pool. PLoS ONE 14(12):e0226394. https://doi.org/10.1371/journ al.pone.0226394

13. Kennedy R, Clifford S, Burleigh T, Waggoner P, Jewell R, Winter N (2020) The shape of and solutions to the MTurk quality crisis. Polit Sci Res Methods 8(4):614-629

14. Washington State Department of Labor and Industries (2020) History of Washington State's Minimum Wage. Available at https:// www.lni.wa.gov. Accessed 7 Jan 2020

15. Zapf M, Denham W, Barrera E, Butt Z, Carbray J, Wang C, Linn J, Ujiki M (2013) Patient-centered outcomes after laparoscopic cholecystectomy. Surg Endosc 27(12):4491-4498

16. Eypasch E (2020) Gastrointestinal Quality of Life Index-10 items (GIQLI-10). Available at https://eprovide.mapi-trust.org. Updated December 2020. Accessed 1 Feb 2020

17. Eypasch E, Williams JI, Wood-Dauphinee S, Ure BM, Schmülling C, Neugebauer E, Troidl H (1995) Gastrointestinal Quality of Life Index: development, validation and application of a new instrument. Br J Surg 82(2):216-222

18. Korolija D, Sauerland S, Wood-Dauphinée S, Abbou CC, Eypasch E, Caballero MG, Lumsden MA, Millat B, Monson JR, Nilsson G, Pointner R, Schwenk W, Shamiyeh A, Szold A, Targarona E, Ure B, Neugebauer E, European Association for Endoscopic Surgery (2004) Evaluation of quality of life after laparoscopic surgery: evidence-based guidelines of the European Association for Endoscopic Surgery. Surg Endosc 18(6):879-897

19. Pieterse AH, Finset A (2019) Shared decision making-much studied, much still unknown. Patient Educ Couns 102(11):1946-1948

20. Mak MHW, Chew WL, Junnarkar SP, Woon WWL, Low JK, Huey TCW, Shelat VG (2019) Patient reported outcomes in elective laparoscopic cholecystectomy. Ann Hepatobiliary Pancreat Surg 23(1):20-33
21. Lam WW, Loke AY (2017) Factors and concerns of patients that influence the decision for spinal surgery and implications for practice: a review of literature. Int J Orthop Trauma Nurs 25:11-18

22. Barlow T, Scott P, Thomson L, Griffin D, Realpe A (2018) The decision-making threshold and the factors that affect it: a qualitative study of patients' decision-making in knee replacement surgery. Musculoskelet Care 16(1):3-12

23. Alokozai A, Crijns TJ, Janssen SJ, Van Der Gronde B, Ring D, Sox-Harris A, Kamal RN (2019) Cost in hand surgery: the patient perspective. J Hand Surg Am 44(11):992.e1-992.e26

24. Hawley ST, Jagsi R (2015) Shared decision making in cancer care: does one size fit all? JAMA Oncol 1(1):58-59

25 Luman W, Adams WH, Nixon SN, Mcintyre IM, Hamer-Hodges D, Wilson G, Palmer KR (1996) Incidence of persistent symptoms after laparoscopic cholecystectomy: a prospective study. Gut 39:863-866

26. Wennmacker S, Lamberts M, Gerritsen J, Roukema JA, Westert G, Drenth J, van Laarhoven C (2017) Consistency of patient-reported outcomes after cholecystectomy and their implications on current surgical practice: a prospective multicenter cohort study. Surg Endosc 31(1):215-224

27. Lamberts MP, Den Oudsten BL, Keus F, De Vries J, van Laarhoven CJ, Westert GP, Drenth JP, Roukema JA (2014) Patientreported outcomes of symptomatic cholelithiasis patients following cholecystectomy after at least 5 years of follow-up: a long-term prospective cohort study. Surg Endosc 28(12):3443-3450

28. van Dijk AH, Wennmacker SZ, de Reuver PR, Latenstein CSS, Buyne O, Donkervoort SC, Eijsbouts QAJ, Heisterkamp J, Hof KI, Janssen J, Nieuwenhuijs VB, Schaap HM, Steenvoorde P, Stockmann HBAC, Boerma D, Westert GP, Drenth JPH, Dijkgraaf MGW, Boermeester MA, van Laarhoven CJHM (2019) Restrictive strategy versus usual care for cholecystectomy in patients with gallstones and abdominal pain (SECURE): a multicentre, randomised, parallel-arm, non-inferiority trial. Lancet 393(10188):2322-2330

29. Vetrhus M, Søreide O, Eide GE, Nesvik I, Søndenaa K (2005) Quality of life and pain in patients with acute cholecystitis. Results of a randomized clinical trial. Scand J Surg 94(1):34-39

30. Levay KE, Freese J, Druckman JN (2016) The demographic and political composition of Mechanical Turk samples. SAGE Open. https://doi.org/10.1177/2158244016636433

Publisher's Note Springer Nature remains neutral with regard to jurisdictional claims in published maps and institutional affiliations. 